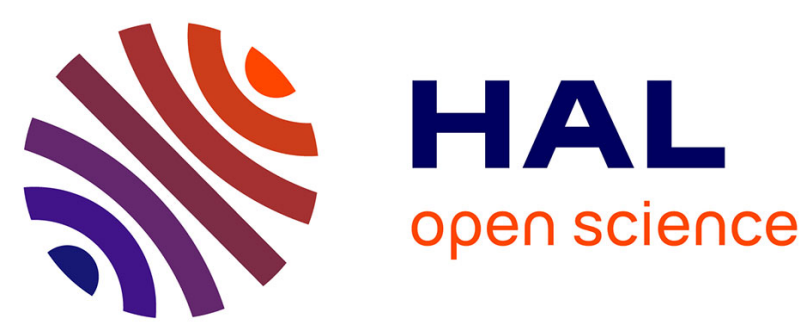

\title{
Optimization of Bifrequential Pump Excitation Parameters by Genetic Algorithm in Nonlinear Vibro Acoustic Wave Modulation Technique
}

Nesrine Houhat, Vincent Tournat, Sébastien Ménigot, Tarek Boutkedjirt, Redouane Drai, Jean Marc Girault

\section{To cite this version:}

Nesrine Houhat, Vincent Tournat, Sébastien Ménigot, Tarek Boutkedjirt, Redouane Drai, et al.. Optimization of Bifrequential Pump Excitation Parameters by Genetic Algorithm in Nonlinear Vibro Acoustic Wave Modulation Technique. 2019 IEEE International Ultrasonic Symposium, Oct 2019, Glasgow, United Kingdom. 10.1109/ULTSYM.2019.8925663 . hal-02311182

\author{
HAL Id: hal-02311182 \\ https://hal.science/hal-02311182
}

Submitted on 10 Oct 2019

HAL is a multi-disciplinary open access archive for the deposit and dissemination of scientific research documents, whether they are published or not. The documents may come from teaching and research institutions in France or abroad, or from public or private research centers.
L'archive ouverte pluridisciplinaire HAL, est destinée au dépôt et à la diffusion de documents scientifiques de niveau recherche, publiés ou non, émanant des établissements d'enseignement et de recherche français ou étrangers, des laboratoires publics ou privés. 


\section{Optimization of Bifrequential Pump Excitation Parameters by Genetic Algorithm in Nonlinear Vibro Acoustic Wave Modulation Technique}

\author{
Nesrine Houhat \\ Research Center in Industrial \\ Technologies CRTI ex CSC \\ Algiers,Algeria \\ n.houhat@crti.dz
}

\author{
Vincent Tournat \\ LAUM, CNRS UMR 6613, \\ Le Mans Université, Le Mans, France \\ vincent.tournat@univ-lemans.fr
}

\author{
Sébastien Ménigot \\ ESEO Group, \\ Angers, France \\ LAUM, CNRS UMR 6613, \\ Le Mans Université, France \\ sebastien.menigot@eseo.fr
}

\author{
Redouane Drai \\ Research Center in Industrial, \\ Technologies CRTI ex CSC \\ Algiers,Algeria \\ r.drai@crti.dz
}

\author{
Jean-Marc Girault \\ ESEO Group, \\ Angers, France \\ LAUM, CNRS UMR 6613, \\ Le Mans Université, Le Mans, France \\ jean-marc.girault@eseo.fr
}

\begin{abstract}
The nonlinear Vibro-Acoustic wave Modulation (VAM) is a widely used technique in nonlinear nondestructive testing. Many research, in this area, are devoted to find the best combination of the excitation parameters, especially the right frequencies to the optimization of the damage detection sensitivity. In a previous study, we have proposed a novel method using the optimal command principle for a VAM system. Indeed, this method has permitted to find automatically the best pump frequency maximizing the nonlinear modulation (NM) effects in a multiple scattering sample. The cost function to be optimized thanks to the pump wave frequency is the correlation coefficient (CC) between a reference output signal without pump and an output modulated probe signal with the presence of the pump wave. In the present work, we aim to improve the nonlinear damage detection by exciting simultaneously two resonance modes of the medium. We consider a new excitation waveform for the pump wave, composed by the sum of 2 frequencies, instead of a monochromatic sine wave. We propose, then, to find automatically the frequencies $f_{p 1}$ and $f_{p 2}$ giving the optimal sensitivity for damage detection. The tested sample is an aluminium bar where nonlinear scatterers can be controllably added or removed. For both $f_{p 1}$ and $f_{p 2}$, the genetic algorithm (GA) seems to stuck on one of the resonance frequencies until it converges to the optimal combination. The corresponding values of the $\mathrm{CC}$ are quite similar to those obtained by a single pump frequency.
\end{abstract}

\section{INTRODUCTION}

The nonlinear Vibro-Acoustic wave Modulation (VAM) is a widely used technique in nonlinear nondestructive testing of materials where the conventional methods reach their limits in the detection of small damage such as a micro-crack. In the VAM tests, the sample is excited simultaneously by a low frequency pump and a higher frequency probing waves [1], [2]. In the presence of damage, the two frequencies are mixed giving rise to new frequency components often referred to as sidebands. Generally, the pump frequency is chosen to correspond to one of the vibration modes of the studied sample in order to amplify the vibration response [2]-[4]. A preliminary modal analysis is, then, necessary for identifying and selecting the resonance frequencies of the sample. It is important to underline that such a procedure requires a further experimental setup and time. Furthermore, a possible drawback of a modal excitation is that the crack or the damaged zone can be located at a strain node of the pump or probe wave, which compromises the generation of the nonlinear modulation.

Many efforts in this area are devoted to find the best combination of the excitation parameters, especially the right frequencies, for the optimization of the VAM sensitivity [4], [5]. In a previous study [6], we have presented a combination of the optimal command [7]-[9] and the VAM technique. A closed loop VAM system able to find automatically the optimal pump frequency that maximizes the nonlinear modulation effects has been presented. The correlation coefficient between a reference output probe signal without the pumping wave and an output modulated probe signal with a pumping wave was considered as the cost function. The main outcome was the existence of optimal pump frequency, corresponding to a one resonance frequency among several resonance frequencies of the sample. This study has permitted to obtain a suboptimal solution by fixing the pump excitation waveform.

The main idea of the present study, is to attempt to improve the previous results by exciting the sample at more of one of his optimal resonance frequencies. Since the medium present several resonance frequencies, we suppose that it should amplify the nonlinear modulation effects between the probe and the pump waves. We propose to test the simplest case; 
those of exciting simultaneously two resonance frequencies of the sample. A novel suboptimal pump excitation waveform is, then, considered. It is a sinusoidal bifrequential signal composed from 2 frequencies $f_{p 1}$ and $f_{p 2}$. An experimental modal analysis is performed to identify the resonance modes of the sample. The two continuous sine pump waves are generated simultaneously with the probe wave. Experimental configurations including different positions and amount of localized damage have been tested.

\section{VAM SYSTEM FOR BIFREQUENTIAL PUMP OPTIMIZATION}

The proposed method is an optimal command method using a closed loop, in order to optimize the VAM sensitivity of crack detection. It makes it possible to find the pump frequency which optimizes the nonlinear modulation effects. The cost function and its parameters need to be adequately chosen. The correlation coefficient $\rho$ between the received probe coda signal without pumping and with the pump excitation constitutes our cost function. With the presence of a nonlinear scatterer in the sample, the probe signal is expected to be modulated by the pump excitation which induces to a decorrelation between the two signals. A correlation coefficient equal to unity means that there is no influence of the pump on the probe wave, i.e., no nonlinear modulation effect in the sample. On the contrary, for the same pump amplitude value, a deviation of $\rho$ from 1 indicates the presence of nonlinear damage leading to a nonlinear modulation effect. In a theoretical point of view, the problem consists in calculating:

$$
\left[f_{p_{1}}^{*}, f_{p_{2}}^{*}\right]=\arg \min _{f_{p 1}, f_{p 2}}\left(\rho\left(f_{p 1}, f_{p 2}\right)\right) .
$$

During the optimization process, for each iteration $k$, the same probe excitation signal $x_{s}(t)$ is transmitted to the sample without the pumping signal, and the received reference probe coda signal $y_{r, k}(t)$ is recorded for a next use. In a second time, a pump excitation $x_{p, k}(t)=A\left(\sin \left(2 \pi f_{p 1, k} t\right)+\right.$ $\left.\sin \left(2 \pi f_{p 2, k} t\right)\right)$ at a frequencies $f_{p 1, k}$ and $f_{p 2, k}$ is transmitted simultaneously with the probe excitation to the medium. The modulated coda probe signal $y_{m, k}(t)$ is also recorded. The added feedback consists in evaluating and optimizing the CC [6]. This coefficient quantifies the resemblance between the reference probe signal and the modulated probe signal by the pump signal. The higher the nonlinear modulation between the probe and the pump signals is, the lower the value of the correlation coefficient is.

The genetic algorithm is used to find a new pumping frequencies $f_{p 1, k+1}$ and $f_{p 2, k+1}$ minimizing the $\mathrm{CC}$ [10]. The frequencies are then modified and all the process described above is reiterated until the algorithm converges toward the best solutions. The first step (called generation 1) consists of choosing randomly $\mathrm{N}$ pumping frequencies couples from a uniform distribution on a given frequencies interval. In our case, we have chosen $10 \mathrm{~Hz} \leq f_{p 1, p 2} \leq 900 \mathrm{~Hz}$. This choice

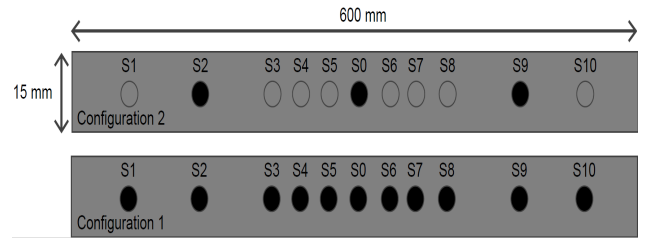

Fig. 1. Specimen schemes of the two configurations. One Aluminium bar $(600 \mathrm{~mm} \times 15 \mathrm{~mm} \times 3 \mathrm{~mm})$ containing 10 tapped holes. Config 1: all the screws are placed on the bar. Config 2: 2 screws are placed at S2 and S9.

is directly related to the experimental setup. The correlation coefficient is evaluated for each pumping frequencies couple and sorted in descending order. To prepare the next step, the $N / 2$ best pumping frequencies couples that minimize the correlation coefficient are kept for the next generation $k+1$ and become parents. N/2 new pumping frequencies couples named offspring are generated by the crossover operator by mixing the best previous pump frequencies couple with one of the remaining better couples. Finally, $40 \%$ of the pumping frequencies couples were mutated to obtain a robust optimization. In our case the population size $\mathrm{N}=6$.

\section{MATERIAL}

\section{A. Specimen Description}

An aluminium bar $(600 \mathrm{~mm} \times 15 \mathrm{~mm} \times 3 \mathrm{~mm})$, with density $\rho=2700 \mathrm{~kg} / \mathrm{m}^{3}$, Poisson ratio $\sigma=0.33$, and Young modulus $\mathrm{E}=69 \mathrm{GPa}$, is used as the specimen for our experiments. The probe wave propagation velocity in the bulk of the bar is estimated to $4838 \mathrm{~m} / \mathrm{s}$. 10 tapped holes with a $4 \mathrm{~mm}$ diameter and localized at distances of $10 \mathrm{~mm}, 30 \mathrm{~mm}, 50 \mathrm{~mm}, 100$ $\mathrm{mm}$ and $200 \mathrm{~mm}$ from each sides of the bar center are drilled. Identical screws can be placed in the tapped holes to mimic nonlinear solid contacts (cracks) such as in [11]. These holes constitute linear scatterers when no screw is present. According to the number of nonlinear scatterers on the bar, different levels of "effective" damage can be obtained. In the present study, two configurations have been more particularly studied: the first one (Config 1) corresponds to the case where all screws are placed (Fig. 1). Configuration 2 (Config 2) corresponds to the case where only 2 screws are positioned at S2 and S9. Note that the nonlinearity level in the sample depends on the screw number and the nuts tightening.

\section{B. Experimental setup}

The closed loop pump frequency optimization process requires the experimental setup depicted in Fig. 2. $250 \mathrm{kHz}$ central- frequencies for transmitting and receiving the probe signal are glued to the ends of the sample. For the transmission of the probe signal, a $100 \mathrm{mVpp}$ sinusoidal burst of 3 periods of $250 \mathrm{kHz}$ frequency, repeated every $20 \mathrm{~ms}$, was transmitted by a function generator (AFG3022, Tektronix, Beaverton, Oregon, USA) and amplified to $60 \mathrm{~dB}(100 \mathrm{Vpp})$ by a power amplifier (Type 2713, Bruël \& Kjær, Nærum, Danemark). Simultaneously, two lower frequencies $f_{p 1}$ and $f_{p 2}$ continuous sine pump signals are generated by the computer-controlled 


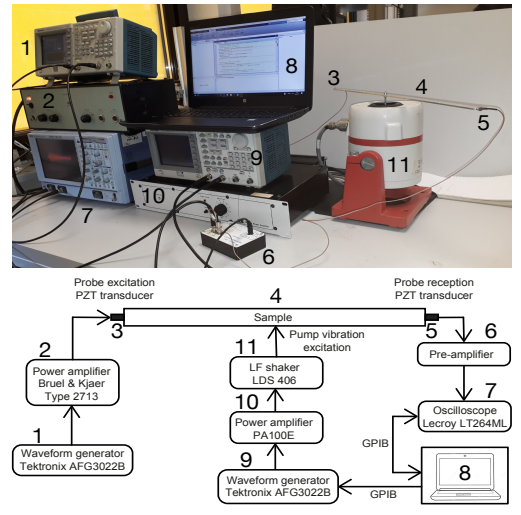

Fig. 2. Experimental setup for the closed loop Vibro-Acoustic Modulation (VAM) technique.

function generator (Tektronix, AFG3022B, Beaverton, Oregon, USA) to change the excitation frequencies during the closedloop optimization process. The pump signals are amplified by a power amplifier (PA100E, Bruël \& Kjær, Nærum, Danemark), and transmitted to the shaker (LDS V406, Bruël \& Kjær, Nærum, Danemark) which is connected to the center of the sample by a screw S0 (Fig. 1). The coda probe signal is detected by the receiving transducer and amplified by a preamplifier (Ciprian, Saint ISMIER, France), then, transmitted to an oscilloscope (LT 264ML, Lecroy, Chestnut Ridge, NY, USA). In order to improve the signal to noise ratio, an average of 300 successive acquisitions is carried out, and a coda averaged signal is recorded. Each measurement lasts about $10 \mathrm{~s}$. Both the function generator and the oscilloscope are controlled by MATLAB (Mathworks, Natick, MA, USA). The pump signal is desynchronized from the probe so that the nonlinear effects are correctly distributed over the successive acquisitions and averaged over the acquired signal.

\section{EXPERIMENTAL RESULTS}

\section{A. Empirical optimization: Correlation coefficient dependency on the pump frequency}

Figs. 3a and 3c show the experimental Frequency Response Functions (FRFs) obtained for Configs 2 and 3, respectively, in a frequency range from $10 \mathrm{~Hz}$ to $900 \mathrm{~Hz}$ with a step of 1 $\mathrm{Hz}$. We can see that the magnitude of the resonance peaks and the corresponding frequencies are different for the two configurations. The results show that there is a shift in the natural frequencies and an overall change in the frequency response due to the effect of the added screws. The first experiment so called "empirical optimization" is to check the existence of global or local minima for the correlation coefficient $v s$. the pump frequency. The prominent observation is the presence of local minima peaks localized at specific vibration frequencies corresponding well to the resonances of the bar, and $\rho \simeq 1$ elsewhere. As illustrated in Fig. 3, the pump frequencies that give minima peaks of $\rho$ coincide well with the resonance frequencies of the sample for the Configs 1 and 2. This confirms the expected effects that at
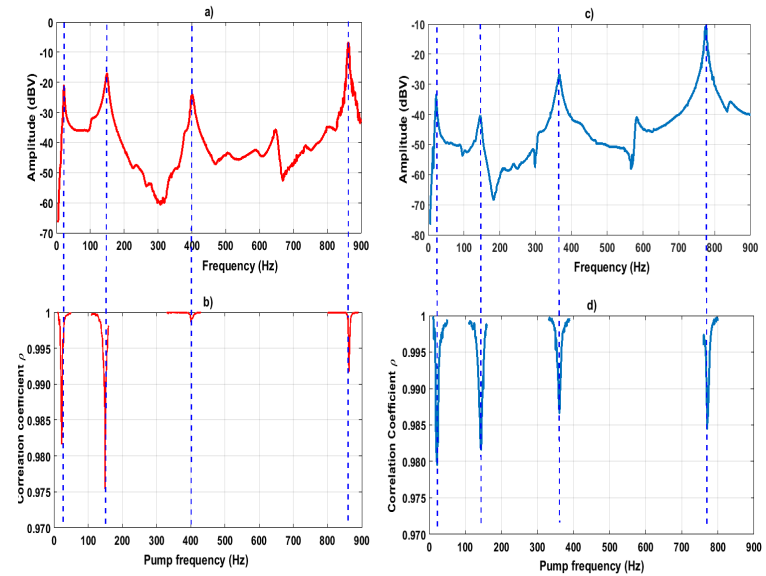

Fig. 3. Comparison between the resonance frequencies obtained from the experimental Frequency Response Function of the Config 2 (a) and the Config 1 (c). Experimental results of correlation coefficient $v s$. pump frequency at the sample resonance frequencies areas for the two sample configurations: b) Config 2 and d) Config 1. The FRF peaks coincide with the minimum peaks of the correlation coefficient $v s$. pumping frequency.

a resonance frequency of the sample, the vibration amplitude is naturally amplified by constructive interferences, leading to a more efficient nonlinear modulation effect.

\section{B. Optimal bifrequential pump excitation}

Figs. 4 and 5 show the genetic algorithm optimization results of the pump frequencies for the Config 1 and Config 2 respectively. Table I shows the accurate pump frequency optimization values. The results of the monochromatic pump optimization are also depicted for a comparison purpose. For the Config 1, it can be noticed that the GA converges to $f_{p 1, o p t}=770 \mathrm{~Hz}$ and $f_{p 2, o p t}=145 \mathrm{~Hz}$ after the $14^{\text {th }}$ generation with $\rho_{o p t}=0.983$. For the monochromatic pump excitation, the GA converges to the global minimum of the empirical cost function at $f_{p, o p t}=22 \mathrm{~Hz}$ (Fig 4.a). For the Config 2, we can see in Fig. 5.a that the GA converges aften the $20^{t h}$ generation, to $f_{p 1, o p t}=149 \mathrm{~Hz}$ and $f_{p 2, o p t}=150 \mathrm{~Hz}$ with a corresponding correlation coefficient $\rho_{o p t}=0.994$ (Fig 5.b). The results of the monochromatic pump optimization obtained previously [6] are also depicted in Fig. 5. It can be noticed that the the optimal pump frequency $f_{p, o p t}=153 \mathrm{~Hz}$ is very close to $f_{p 1, o p t}$ and $f_{p 2, o p t}$ and to the optimal frequency obtained by the empirical optimization $\left(f_{p, E O}=150 \mathrm{~Hz}\right)$. Fig 5.b shows that the $\mathrm{CC}$ value obtained for the monochromatic pump is quite similar then those obtained for the bifrequential pump excitation. The GA converges to the global minimum of the empirical cost function (Fig 3.b), for both $f_{p 1}$ and $f_{p 2}$, in a pump frequency range between 10 and $900 \mathrm{~Hz}$, including four (4) local minima of the cost function. Moreover, until the optimization process, the GA stucks on values very close to the resonance frequencies of the sample. We can see clearly, in Fig 4.a, that $f_{p 1}=731 \mathrm{~Hz}$ at the first generation. It is equal to $174 \mathrm{~Hz}$ from generations 2 to 4, then $365 \mathrm{~Hz}$ until generation 12. Finally, it reaches the optimal frequency at $=770 \mathrm{~Hz}$. 


\begin{tabular}{l|cccc|cccc}
\hline & \multicolumn{4}{|c|}{ Config 1 } & \multicolumn{3}{c}{ Config 2 } \\
\hline & $f_{p, E O}(\mathrm{~Hz})$ & $\left.f_{p, o p t} \mathbf{( H z}\right)$ & $f_{p 1, o p t}(\mathbf{H z})$ & $f_{p 2, o p t}(\mathbf{H z})$ & $f_{p, E O}(\mathrm{~Hz})$ & $f_{p, o p t}(\mathbf{H z})$ & $f_{p 1, o p t}(\mathbf{H z})$ & $f_{p 2, o p t}(\mathbf{H z})$ \\
\hline Values & 22 & 22 & 770 & 145 & 150 & 153 & 150 & 149 \\
\hline
\end{tabular}

THE PUMP FREQUENCIES OBTAINED FROM THE MONOCHROMATIC AND THE BIFREQUENTIAL PUMP OPTIMIZATION, AND THE GLOBAL MINIMUM OF THE EMPIRICAL COST FUNCTION $f_{p, E O}$.
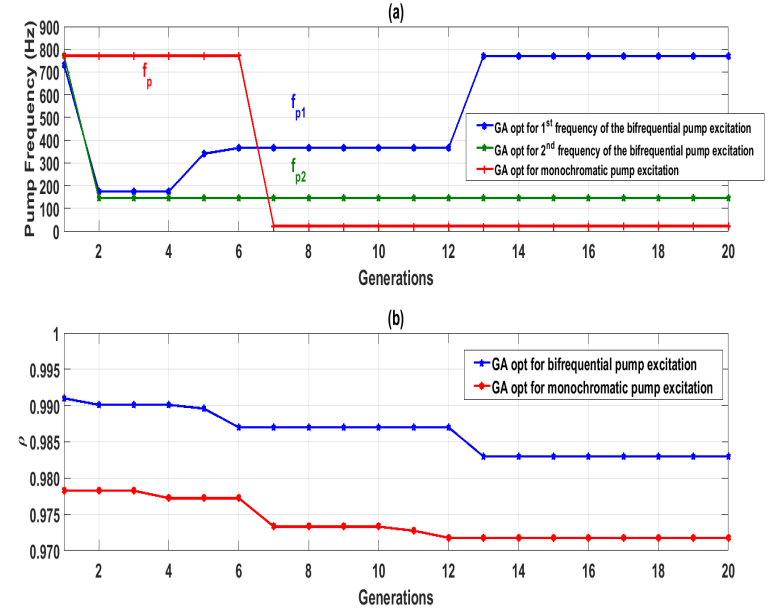

Fig. 4. Genetic algorithm (GA) optimization results for the Config 1: (a) The pump frequencies $v s$. generations. The GA converges to $f_{p, o p t}=22 \mathrm{~Hz}$ after the $7^{\text {th }}$ generation for the monochromatic pump. For the bifrequential pump, $f_{p 1, o p t}=145 \mathrm{~Hz}$ and $f_{p 2, o p t}=770 \mathrm{~Hz}$. (b) The corresponding correlation coefficient $\rho v s$. generations for the bifrequential and the monochromatic pump excitation.

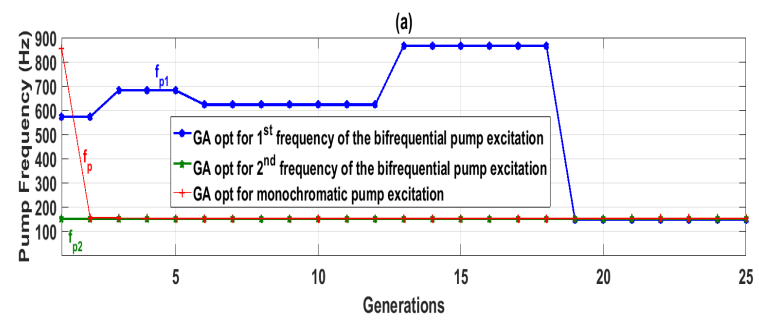

(b)

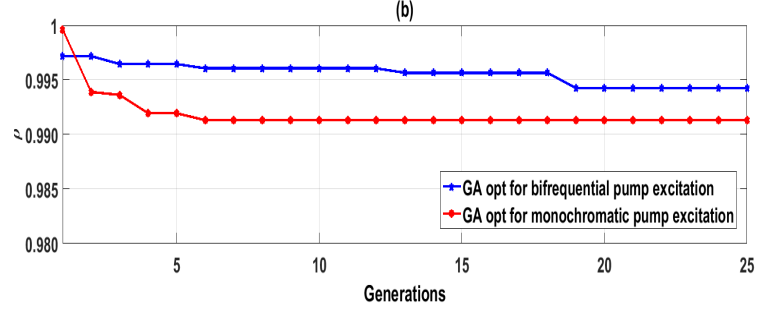

Fig. 5. Genetic algorithm (GA) optimization results for the Config 2: (a) The pump frequencies $v s$. generations. The GA converges to $f_{p, o p t}=153 \mathrm{~Hz}$ after the $4^{\text {th }}$ generation for the monochromatic pump. For the bifrequential pump, $f_{p 1, \text { opt }}=149 \mathrm{~Hz}$ and $f_{p 2, \text { opt }}=150 \mathrm{~Hz}$. (b) The corresponding correlation coefficient $\rho v s$. generations for the bifrequential and the monochromatic pump excitation.

\section{Discussion AND CONCLUSIONS}

Optimal bifrequential pump excitation was automatically selected in order to maximize the nonlinear modulation effects in VAM tests. Two experimental configurations including different positions and amount of localized damage have been tested. The main result is that the GA seems to propose only a solutions close to the resonance frequencies of the sample, until it converges to the optimal couple. The corresponding values of the $\mathrm{CC}$ are quite lower to those obtained by a single pump frequency. Nevertheless, we have expected that exciting the sample at two optimal pumping frequencies should give rise to a more important de- correlation value. This observation may be due to the GA precision. We think that the $\mathrm{CC}$ value may be refined by an hybridization of the GA with a more accurate algorithm like the Descent Gradient Algorithm. Also, as a perspective, the optimization of the phase between the two sinusoidal pump will be considered. To summarize, an automatic adaptation of a bifrequential pump excitation by a closed loop VAM system giving the optimal damage detection is proposed. The optimal pumping frequencies corresponded to a resonance frequencies of the studied sample.

\section{REFERENCES}

[1] D. M. Donskoy and A. M. Sutin, "Vibro-acoustic modulation nondestructive evaluation technique," Journal of intelligent material systems and structures, vol. 9, no. 9, pp. 765-771, 1998.

[2] L. Pieczonka, A. Klepka, A. Martowicz, and W. J. Staszewski, "Nonlinear vibroacoustic wave modulations for structural damage detection: an overview," Optical Engineering, vol. 55, no. 1, p. 011005, 2015.

[3] L. Pieczonka, A. Klepka, M. Adamczyk, W. Staszewski, F. Aymerich, and T. Uhl, "Optimal selection of parameters for impact damage detection in composites based on nonlinear vibro-acoustics modulations," in ECCM16 16th European Conference on Composite Materials, Seville, Spain, 22-26 June 2014.

[4] B. Liu, Z. Luo, and T. Gang, "Influence of low-frequency parameter changes on nonlinear vibro-acoustic wave modulations used for crack detection," Structural Health Monitoring, p. 1475921716689385, 2017.

[5] K. Dziedziech, L. Pieczonka, M. Adamczyk, A. Klepka, and W. J. Staszewski, "Efficient swept sine chirp excitation in the non-linear vibro-acoustic wave modulation technique used for damage detection," Structural Health Monitoring, vol. 17, no. 3, pp. 565-576, 2018.

[6] N. Houhat, V. Tournat, S. Ménigot, T. Boutkedjirt, and J.-M. Girault, "Optimal pump excitation frequency for improvement of damage detection by nonlinear vibro acoustic modulation method in a multiple scattering sample," Applied Acoustics, vol. 155, pp. 222-231, 2019.

[7] S. Ménigot, J.-M. Girault, I. Voicu, and A. Novell, "Optimization of contrast-to-tissue ratio by frequency adaptation in pulse inversion imaging," IEEE transactions on ultrasonics, ferroelectrics, and frequency control, vol. 59, no. 11, 2012.

[8] S. Ménigot and J.-M. Girault, "Optimization of contrast resolution by genetic algorithm in ultrasound tissue harmonic imaging," Ultrasonics, vol. 71, pp. 231-244, 2016.

[9] N. Houhat, S. Ménigot, T. Boutkedjirt, R. Drai, and J.-M. Girault, "Optimal stochastic excitation for linear flaw detection in a solid material," Lecture Notes in Computer Science, vol. 11401, pp. 229-236, 2019.

[10] R. L. Haupt, S. E. Haupt, and S. E. Haupt, Practical genetic algorithms. Wiley New York, 1998, vol. 2.

[11] J.-B. Legland, Y. Zhang, O. Abraham, O. Durand, and V. Tournat, "Evaluation of crack status in a meter-size concrete structure using the ultrasonic nonlinear coda wave interferometry," The Journal of the Acoustical Society of America, vol. 142, no. 4, pp. 2233-2241, 2017. 